\title{
Intelligibility of interrupted meaningful and nonsense speech with and without intervening noise
}

\author{
J. VERSCHUURE and M. P. BROCAAR \\ Erasmus University Rotterdam, Rotterdam, The Netherlands
}

\begin{abstract}
The insertion of noise in the silent intervals of interrupted speech has a very striking perceptual effect if a certain signal-to-noise ratio is used. Conflicting reports have been publiohed as to whether the inserted noise improves speech intelligibility or not. The major difference between studies was the level of redundancy in the speech material. We show in the present paper that the noise leads to a better intelligibility of interrupted speech. The redundancy level determines the possible amount of improvement. The consequences of our findings are discussed in relation to euch phenomena as continuity perception and pulsation threshold measurement. $A$ hypothesis is formulated for the processing of interrupted stimuli with and without intervening noise; for otimuli presented with intervening noise, the presence in the auditory system of an automatic interpolation mechanism is aseumed. The mechanism operates only if the noise makes it impossible to perceive the interruption.
\end{abstract}

The insertion of broadband noise in the silent gaps of interrupted speech has a very striking overall auditory effect. While the interrupted speech sounds hoarse and raucous, the insertion of the noise restores fluency: the listener has the impression that he is hearing continuous speech with a superimposed pulsating noise. This phenomenon was first described by Miller and Licklider in 1950 . Whether or not the added noise does lead to a real increase in speech intelligibility has been a matter of discussion ever since. This debate is continued in the present paper.

We became interested in the phenomenon after we had utilized the pulsation threshold technique (Houtgast, 1972, 1974) for the measurement of frequency selectivity (Verschuure, 1977, 1978, 1981a, $1981 \mathrm{~b})$. The technique is based on the finding that an alternation of two sine waves can be perceived as a superposition of one pulsating and one continuous tone, given certain relations between frequencies and levels of the alternating sine waves. The perception of one tone as if it were continuous means that somewhere in the auditory system a gap in information is filled up.

With interrupted speech and intervening noise, the gap in information must affect the intelligibility of

The authors would like to thank all those who gave so willingly of their time to play the part of listener and S. Smit-Hermer for reading the speech material, $G$. van den Brink for stimulating discussions both during and after the investigation and during the preparation of the paper. Also, the financial support received from the Heinsius Houbolt Fund is gratefully acknowledged. The authors' mailing address is: Department of Otolaryngology, Erasmus University Rotterdam, P.O. Box 1738, 3000 DR Rotterdam, The Netherlunds. the speech. Since speech with gaps means that parts of the information to be conveyed are physically missing, perception of such speech as being continuous implies that the physically absent parts of the speech are filled in by some process. Using interrupted speech without intervening noise means that we present the observer with the same speech information, but now in a different context. The lack of fluency forces the listener to guess at what might have been said. A difference in an intelligibility score between the two conditions would mean that two different kinds of filling-in processes were involved.

This study investigates whether the filling-in processes with and without intervening noise are different and whether the processes depend on the redundancy level of the speech material. The relationship with continuity perception will be discussed.

\section{Previous Investigations}

Most experiments on interrupted speech have been inspired by the finding of Cherry (1953) and Cherry and Taylor (1954) that speech alternated between the ears is less intelligible at a rate of about $4 \mathrm{~Hz}$ than at lower or higher rates. For this reason, although this paper is mainly on interrupted speech, we will describe some findings on alternated speech. We must clearly distinguish between interrupted speech and alternated speech. In the former case, parts of the speech are missing; in the latter case (as investigated by most authors), all speech information is available to the listener, but it is split up between the two ears. A third kind of speech with characteristics between those of interrupted and alternated speech is "segmented" speech (Huggins, 1975a, 1975b), in which all speech information presented to 
the listener is segmented into parts that are separated from one another by silent intervals.

It can be shown that minimum intelligibility of alternated speech is found at an alternation rate of about $4 \mathrm{~Hz}$. Its value depends on the speaking rate (Huggins, 1964; Speaks \& Trooien, 1974; Wingfield \& Wheale, 1975), on the redundancy level of the speech (Hopkinson, 1967; Kreul, 1971; Wingfield \& Wheale, 1975), on the time relation between the alternations and the syllables (Hughes, House, Goldstein, \& Rupf, 1966), and probably on the measuring technique (Hopkinson, 1967; Speaks \& Trooien, 1974).

Schubert and Parker (1955) inserted noise in the silent intervals of alternated speech to mask possible cross-talk and found that intelligibility was improved when noise was added. Hopkinson (1967) and Kreul (1971) could not corroborate this finding. The major difference between their experimental techniques and those of Schubert and Parker was that they used monosyllables or nonsense speech and Schubert and Parker used running speech. They found that the noise, if it had any effect, lowered speech intelligibility. They further suggested that the measuring technique ("speech shadowing") used by Schubert and Parker had flaws which might account for the low intelligibility of alternated speech measured by these authors.

One point all the above authors agreed on was the wide variability between listeners.

We may conclude that the effect of inserted noise on the intelligibility of alternated speech is still unclear. The data suggest that either a difference in procedure or a difference in speech material (redundancy level?) is responsible for the apparently conflicting results.

The focus of the present paper-intelligibility of interrupted speech-is a result of the fact that conditions of pulsation threshold measurement involve interruptions. Parts of the speech are actually missing.

Miller and Licklider (1950) were the first to measure the intelligibility of interrupted speech, using phoneme-balanced lists of monosyllables. They found that intervening noise had a striking perceptual effect on interrupted speech, which seemed to become more intelligible with intervening noise. Actual measurement of intelligibility showed, however, that, at rates below $10 \mathrm{~Hz}$, intelligibility of interrupted speech was the same with noise as it was without, and that, at higher interruption rates, it was lower with noise than it was without. The noise, if it had any effect, lowered speech intelligibility. Dirks and Bower (1970) argued that this reduction was caused by masking effects.

Powers and Speaks (1973) showed that intelligibility was lowest at interruption rates of about $1 \mathrm{~Hz}$. Its value depended on rate of speaking and on the on-off time ratio; the minimum score did not depend on the rate of speaking, given a certain on-off ratio.
Cherry and Wiley (1967) found that they could make continuous speech almost unintelligible if all low-energy parts of the speech were replaced by silent intervals. If white noise was inserted in the intervals, the speech regained its intelligibility almost completely; this process depended rather critically on the signal-to-noise ratio of interrupted speech and intervening noise.

Powers and Wilcox (1977) found that the intervening noise improved intelligibility of regularly interrupted speech also. They used test sentences and showed that the amount of improvement depended on the signal-to-noise ratio, the best intelligibility being achieved at ratios between -6 and $-24 \mathrm{~dB}$.

Miller and Licklider's results conflict with those of Cherry and Wiley and those of Powers and Wilcox. The major difference between the experiments is the speech material used. It suggests that the greater redundancy level of sentences, as compared with monosyllables, could be the main reason for the observed difference.

Surveying the literature, we see that contradictory results have been reported on the influence of noise on the intelligibility of interrupted speech. Similar contradictions are found in reports on alternated speech. The major difference between authors reporting conflicting results is the speech material used, in particular its redundancy level. Therefore, and because we would like to find a basis for the interpretation of pulsation threshold data, we decided to investigate the effect of the redundancy level on the intelligibility of interrupted speech with and without intervening noise.

\section{METHOD}

We used two kinds of speech in our experiments, both consisting of short sentences with correct grammatical structure. The first kind ("meaningful speech") consisted of normal sentences with a clear meaning, for example, "All bottles are in the cupboard." The second kind ("nonsense speech") consisted of strings of words without meaning (but with correct grammatical structure), for example, "The church bell drank a sailor." All speech was in Dutch.

The sentences were recorded on tape on a two-track reel recorder (Revox A 77). They were read by a trained female speaker from our phoniatric department. She was seated in an IAC acoustic room, and was asked to read at as constant a level as possible. The average level was adjusted to a $-3-\mathrm{dB}$ reading on the VU-meter of the recorder, which gives a peak reading of about $0 \mathrm{~dB}$. $\mathrm{A} 1-\mathrm{kHz}$ calibration tone at a level of $0 \mathrm{~dB}$ was recorded on the tape before the recorded speech.

The sentences were rearranged and presented with two lists of nonsense speech alternating with two lists of meaningful speech, and so on. Each list consisted of 10 sentences. Eight lists of meaningful speech and eight lists of nonsense speech were constructed.

Interruptions were inserted as appropriate with the aid of our pulsation threshold equipment (described by Verschuure, Rodenburg, \& Maas, 1976). The speech signal was fed into gate 1 of the equipment. The signal for the second gate was generated by a GR-1382 noise generator set for USASI (speech) noise. The equipment was set to a switching rate of $4 \mathrm{~Hz}$, as in almost all our pulsation experiments. This means that $125 \mathrm{msec}$ of speech was alternated with 125 msec of noise. The switching transients were 
Oaussian with a time constant of $3.55 \mathrm{msec}$. The two signals were recorded on separate channels of another recorder (Revox A 77). The speech was recorded at a level giving a $-15-\mathrm{dB}$ level for the continuous calibration tone. The noise was recorded at a level of $0 \mathrm{~dB}$ for the uninterrupted noise. This method of recording made it possible to replay the speech in the test setting as interrupted speech alone (replay channel 1) or as interrupted speech plus intervening noise at a signal-to-noise ratio of $-15 \mathrm{~dB}$ (parallel replay of channels 1 and 2).

In our experiments, the signals were replayed through a Peters AP-6 audiometer with Grason-Stadler TDH-39 headphones. They were presented monaurally. The calibration tone was used to calibrate the audiometer as customary in speech audiometry. This calibration procedure yields a $100 \%$ intelligibility score, in persons with normal hearing, for normal phoneme-balanced word lists at an intensity level of $40 \mathrm{~dB}$. We presented the signals at $70 \mathrm{~dB}$, which means that the speech was at $55 \mathrm{~dB}, 15 \mathrm{~dB}$ higher than the level for full intelligibility of monosyllables.

All listeners were native speakers of Dutch and had normal hearing. They worked either in the Department of Otolaryngology or the Department of Biological and Medical Physics of Erasmus University Rotterdam. The majority were not familiar with the background of the experiments.

The presentation of each sentence was followed by a 7 -sec silent interval, during which the listeners were asked to repeat the sentence. This method was chosen in order to avoid possible artifacts due to "speech shadowing," as suggested by Hopkinson (1967) and Kreul (1971).

The errors made by the listeners were noted, and the intelligibility score was calculated as the percentage of words correctly understood. For this purpose, articles were ignored and compound words such as "windmill" were treated as two separate words.

The listeners were divided at random into two groups, to whom the lists were presented in different sequences. A total of 16 lists were presented in two sessions (8 lists per session), with the two sessions never being held on the same day. The sequence of presentation for Group 1 was as follows: Session 1 -nonsense speech (NS) without intervening noise, NS with intervening noise; meaningful speech (MS) without noise, MS with noise; NS with noise, NS without noise; MS with noise, MS without noise. Session 2NS with noise, NS without noise; MS with noise, MS without noise; NS without noise, NS with noise; MS without noise, MS with noise. Group 2 received the lists in the same order, except that whenever Group 1 had a list with noise, they had one without noise, and vice versa.

The presentation of the lists in groups of two with the same kind of speech permits direct comparison between speech with and speech without noise. The alternation of nonsense and meaningful speech spreads the influence of increasing experience over the two kinds of speech.

We assessed the possible influence of a learning effect by performing a tentative analysis after the data for the first eight listeners had been collected. We found a marked learning effect for nonsense speech without intervening noise and a much less marked effect for nonsense speech with intervening noise. We therefore preceded the formal presentations by two extra recorded lists of nonsense speech, one without noise and one with noise, so that all listeners had already acquired some experience by the time they started the test proper.

\section{RESULTS}

All 18 observers listened to four lists of each kind of speech with intervening noise and to four lists without intervening noise. A first impression of the effect of noise on intelligibility can be obtained from the Figures 1 and 2. Figure 1 shows the distribution of the 72 intelligibility scores for interrupted mean-

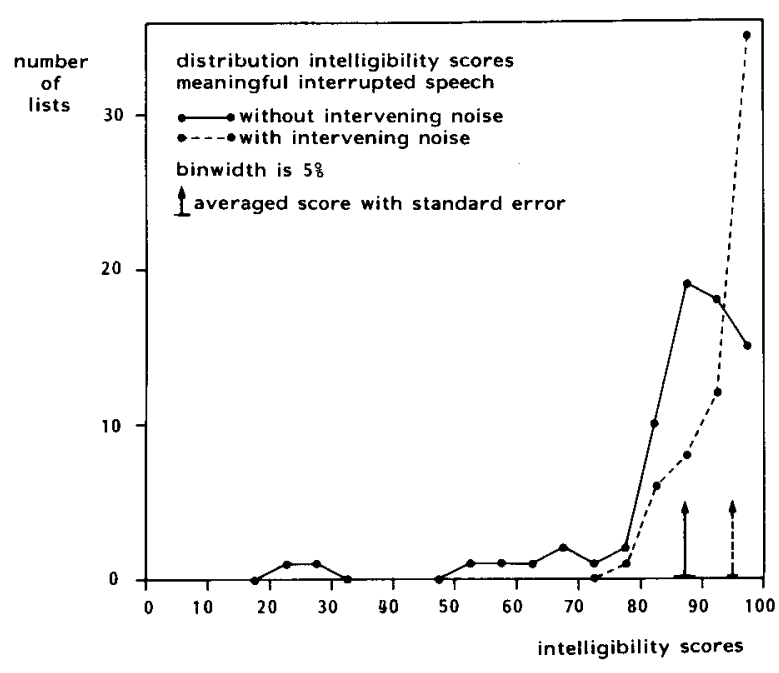

Figure 1. Frequency distributions of Intelliglbllity scores with and without intervening nolse for interrupted meaningful speech. Class interval is $5 \%$, and number of lists that had a score within the specific interval are as given. Arrow shows mean score; horfzontal bar shows the standard error of the mean.

ingful speech with noise and of the 72 scores for that without noise. The class interval is $5 \%$, and the number of lists scored within the interval is given. The average score for all 72 lists is shown by an arrow, and the standard error of the mean by a horizontal bar.

In a similar fashion, Figure 2 shows the results for interrupted nonsense speech.

The figures show that intelligibility of interrupted speech improves for both kinds of speech if the silent intervals are filled with noise. The improvement can be seen in the means and in the distribution pattern itself, although the distributions show a large overlap.

Every listener is represented four times in the figures, which gives dependent scores. We therefore determined the effect of noise on intelligibility for pairs of lists. As mentioned above, the speech was always presented to the listeners in paired lists, one without intervening noise and one with, which permitted assessment of the effect of the intervening noise. We compensated for differences between the lists by presenting the first list of the pair with noise to half the listeners and the same list without noise to the other half.

The results are shown in Table 1. Columns 2, 3, and 4 show the number of listeners that had a better, equal, or worse intelligibility score when noise was added. The comparison shows again the general trend for better intelligibility with intervening noise, although worse scores also are found in some cases.

This brings us to the question of whether the improvement is significant. The distribution functions of Figures 1 and 2 are far from normal, especially 


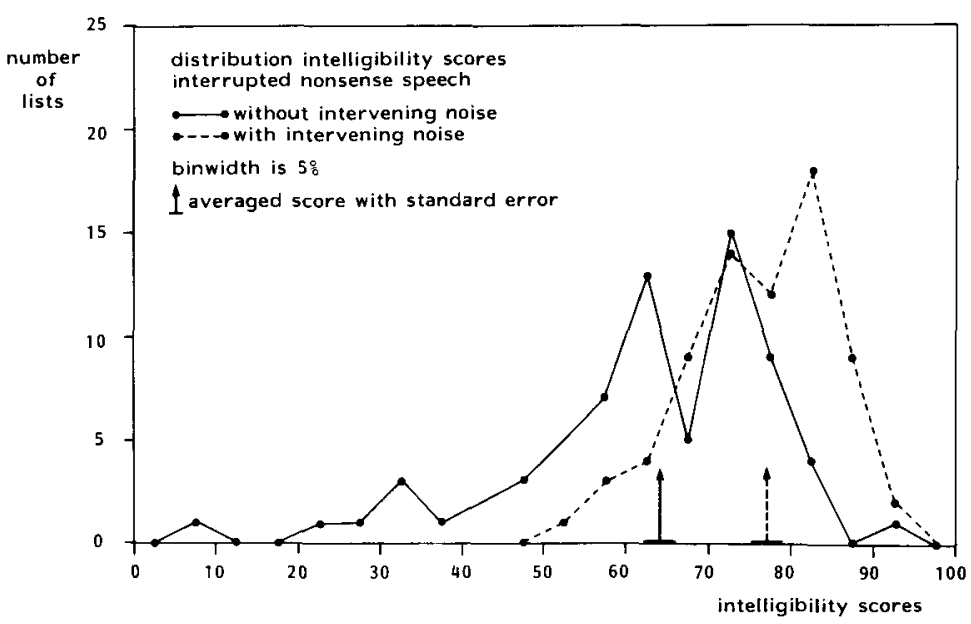

Figure 2. Distributions of intelligibility scores for nonsense speech with and without intervening noise, presented as in Figure 1.

those for meaningful speech in Figure 1. This means that Student's t test or an analysis of variance cannot be used for testing the significance. We therefore applied the Wilcoxon matched-pairs signed-ranks test (Siegel, 1956). This nonparametrical test takes the actual scores into account and does not look only at the direction of change in score. In the test, the scores with and without noise for each pair of lists are rank ordered, and the sum of the ranks without noise are used for the testing. The significance levels thus determined are footnoted below the table. The results presented in Table 1 show that noise significantly improves intelligibility of both kinds of speech for each pair of lists.

We have seen so far that a real improvement is present. Figures 1 and 2 show that there is a great variability in intelligibility scores, in particular for nonsense speech. In order to know where the scores

Table 1

The Effect of Intervening Noise on the Intelligibility of Interrupted Speech for 18 Observers

\begin{tabular}{|c|c|c|c|}
\hline \multirow{2}{*}{$\begin{array}{c}\text { Pair } \\
\text { Number }\end{array}$} & \multicolumn{3}{|c|}{$\begin{array}{l}\text { Number of Listeners Who Reacted to } \\
\text { Addition of Noise by Scoring: }\end{array}$} \\
\hline & Better & Equal & Worse \\
\hline \multicolumn{4}{|c|}{ Nonsense Speech } \\
\hline 1 & 17 & 0 & $1 *$ \\
\hline 2 & 14 & 1 & 3* \\
\hline 3 & 15 & 1 & $2^{*}$ \\
\hline 4 & 14 & 0 & $4 *$ \\
\hline \multicolumn{4}{|c|}{ Meaningful Speech } \\
\hline 1 & 13 & 2 & $3 *$ \\
\hline 2 & 12 & 0 & $6 \dagger$ \\
\hline 3 & 14 & 0 & $4^{*}$ \\
\hline 4 & 13 & 0 & $5 \dagger$ \\
\hline
\end{tabular}

*Significance of improvement (Wilcoxon), $p<.005$.

tSignificance of improvement (Wilcoxon), $p<.025$. of various observers fall within the two distributions and whether the lower and higher tails of the distributions with and without intervening noise are made up of the scores of the same observers, we plotted, in Figure 3 for meaningful speech and in Figure 4 for nonsense speech, the improvement of the intelligibility score as a function of the score of interrupted speech without intervening noise. In these figures, the horizontal broken lines represent no improvement of intelligibility with noise, the vertical broken lines represent the maximum score of $100 \%$ intelligibility, and the oblique broken lines of slope $-45 \mathrm{deg}$ represent the maximum possible improvement of intelligibility up to a score of $100 \%$ when noise is added. The regression line of Figure 3 indicates that intelligibility of interrupted meaningful speech with intervening noise is always close to $95 \%$, although intelligibility without noise can be very different for different listeners. The noise seems to induce a large improvement of intelligibility for those who perform poorly without intervening noise, but it does not do much for those who perform well without noise. All observers showing worse intelligibility with intervening noise had an intelligibility score without intervening noise of $90 \%$ or more. The tails of the distributions with and without intervening noise are, therefore, not made up of the scores of the same observers. The two distributions are not correlated.

The regression line of Figure 4 for nonsense speech shows the same general trend as that of Figure 3. In this case, intervening noise improves the score up to about $77 \%$. However, the regression line has a slope of less than 1.0, which implies that observers scoring relatively low without intervening noise do the same with intervening noise. This means that the scores of the same observers are found in the lowscore tails of the two distributions, which are correlated. 


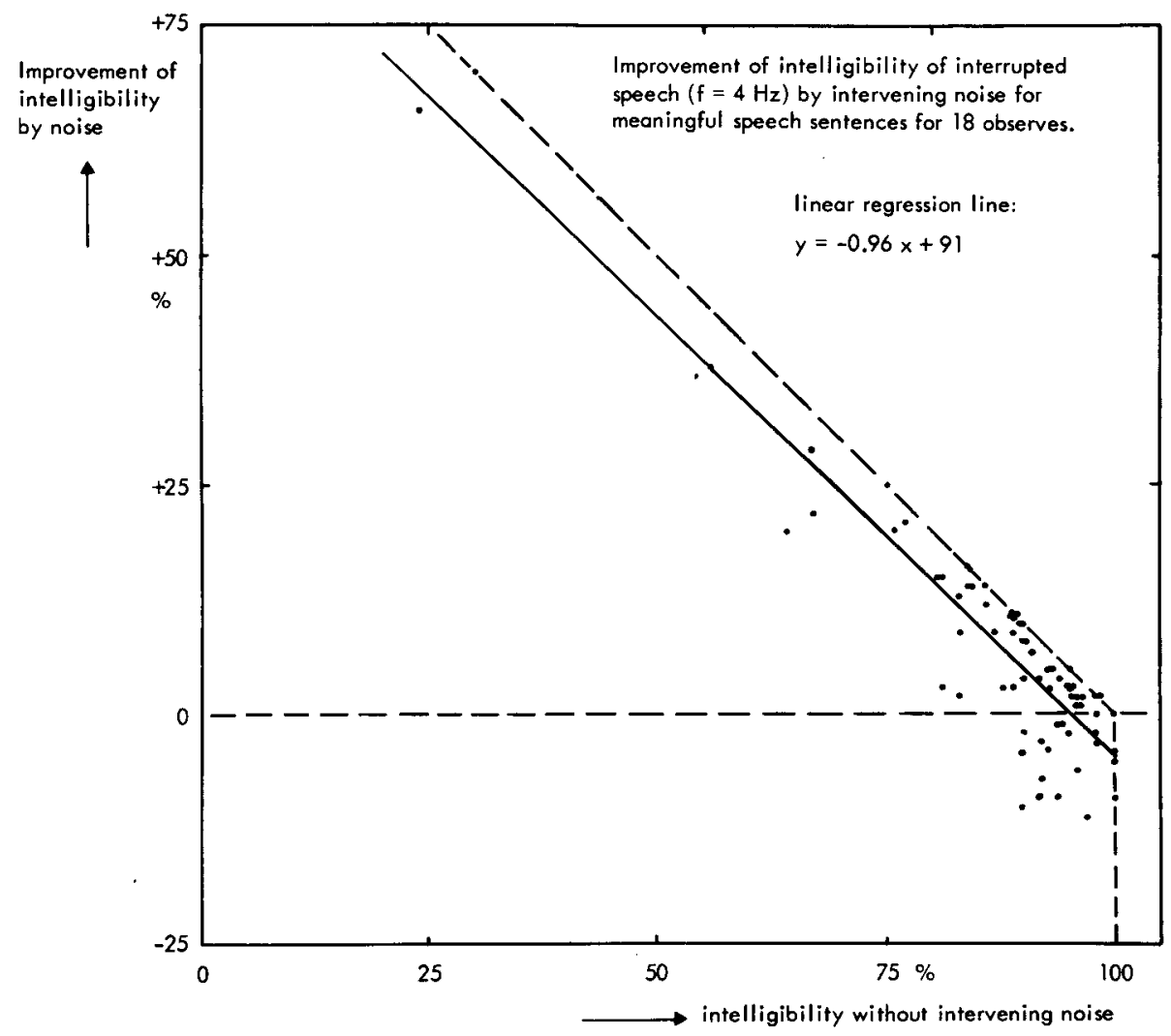

Figure 3. Correlogram of improvement in intelliglbility of interrupted meaningful speech by Intervening nolse as a function of the intelliglblity of the same speech without noise.

\section{DISCUSSION}

\section{Speech Materlal}

In Table 2, using approximate intelligibility scores, we compare our speech material with that of other investigators of interrupted speech.

All authors agree that lowest intelligibility is found at interruption rates between 1 and $2 \mathrm{~Hz}$. Their minimum scores are shown in the first row. We preferred to use a rate of $4 \mathrm{~Hz}$ in order to be able to apply our results and interpretations to pulsation threshold data. For the same reason, we chose to use an on-off ratio of 0.50 like most authors did. Intelligibility scores for these conditions (sometimes interpolated and sometimes extrapolated) are shown in the second row if no intervening noise is presented and in the third row if intervening noise is presented. We see that our scores for nonsense speech without noise are comparable to the results of Miller and Licklider for the less experienced listeners. The scores with intervening noise are fully comparable.

Our scores for meaningful speech are a bit better than those of Powers and Wilcox. The difference may be due to the speaking rate which in our material was deliberately low (about 138 words per minute, wpm) and the same for meaningful and nonsense speech. Powers and Speaks have shown that speaking rate is an important factor for intelligibility at $4 \mathrm{~Hz}$. Using connected discourse, they found a score of $70 \%$ for a rate of $216 \mathrm{wpm}$ and one of $90 \%$ for $186 \mathrm{wpm}$. Our score was obtained with sentences spoken at a much lower rate, and the score was near their maximum score.

We conclude that our speech material is comparable to that of others. The differences in scores for meaningful and nonsense speech are due to the redundancy level of the speech, nonsense speech being comparable to monosyllables and meaningful speech to connected discourse.

We want to point out that our nonsense sentences presented normally or as alternated speech at $2,4,6$, and $8 \mathrm{~Hz}$ always resulted in a $100 \%$ intelligibility score, contrary to the findings of Schubert and Parker (1955) and also to those of Marks and Miller (1964), who showed a maximum intelligibility for this kind of material (anomalous sentences) of $80 \%$. Our experience with alternated nonsense speech is in line with that of Bocca and Calearo (1963), who used alternated speech to differentiate between pathologies of the central auditory pathways.

So far, our considerations have been based on the assumption that all lists of meaningful speech have 


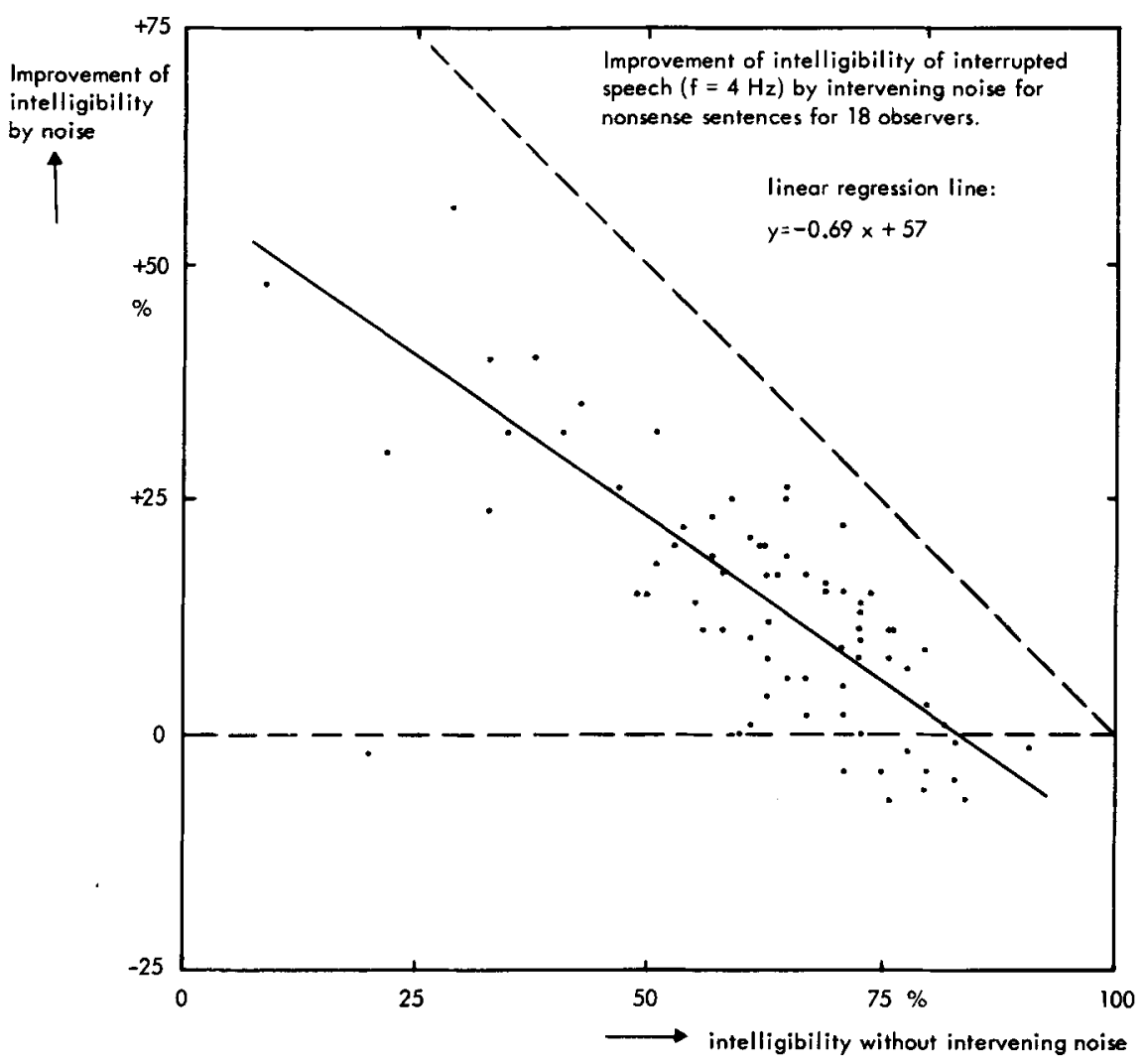

Figure 4. Correlogram of improvement of intelligibility of interrupted nonsense speech by intervening noise as a function of the intelligiblitty of the same speech without noise.

the same intrinsic difficulty and that all lists of nonsense speech have the same intrinsic difficulty (doubtlessly higher). However, thanks to the fact that our 18 listeners were divided into two groups of equal size, we do have a means of assessing the variation in intrinsic difficulty between the lists. It should be stressed that the two lists in a given pair are exactly the same for all listeners; however, if we call the two lists of, say, Pair $44 a$ and $4 b$, then half of the listeners heard $4 a$ with noise and $4 b$ without, while the other half heard $4 a$ without noise and $4 b$ with. This means that we do, in fact, have data on the intelligibility of all 16 lists both with and without noise. In this respect, it is no use to compare the intrinsic intelligibility of, say, Pairs 1 and 3 , since the dif- ference found involves a learning effect the precise magnitude of which is not known. However, we can meaningfully compare the intrinsic difficulty of the two lists within each pair. Application of MannWhitney's $\mathrm{U}$ test (taking $\mathrm{p}<.05$ as the significance level) shows that the two lists of Pair 3 for meaningful speech and the two lists of Pair 3 for nonsense speech, all presented with noise, are the only lists between which there is a significant difference. This difference can be seen in regression lines like those in Figures 2 and 3, but plotted and computed separately for each pair. In both cases, for both meaningful and nonsense speech, the regression lines are parallel, but the averaged scores with noise are $89 \%$ and $98 \%$, respectively, for meaningful speech (standard errors

Table 2

Mean Intelligibility Scores of Interrupted Speech

\begin{tabular}{lccccc}
\hline & \multicolumn{3}{c}{ Authors and Materials } \\
\cline { 3 - 5 } & $\begin{array}{c}\text { Miller \& Licklider } \\
\text { Monosyllables }\end{array}$ & $\begin{array}{c}\text { Powers \& Speaks } \\
\text { Connected Discourse }\end{array}$ & $\begin{array}{c}\text { Powers \& Wilcox } \\
\text { Sentences CID }\end{array}$ & $\begin{array}{c}\text { Verschuure \& Brocaar } \\
\text { Nonsense }\end{array}$ \\
\hline Minimum & 40 & 60 & 55 & 64 & Meaningful \\
At 4 Hz & $50-80$ & $70-90$ & 80 & 87 \\
4-Hz Noise & 79 & & 85 & 95 \\
\hline
\end{tabular}

Note-At $4 \mathrm{~Hz}$, the value for monosyllables (Miller \& Licklider) depends on the experience of the listeners; that for connected discourse (Powers \& Speaks) depends on the speaking rate. 
of $1 \%$ and $2 \%$ for $\mathrm{n}=9$ ) and $67 \%$ and $78 \%$, respectively, for nonsense speech (standard errors of $3 \%$ for $n=9$ ). We conclude that differences between the lists are minor and did not influence the results.

A further point of interest is the difference in slope of the regression lines in Figures 3 and 4. It might be argued that the difference is due to a higher learning effect for nonsense speech. All data were examined for a learning effect. We considered a learning effect to be present when the score for List Pair 2 was higher than that for List Pair 1 and the score for List Pair 4 was higher than that for List Pair 3. The analysis showed that for nonsense speech without noise, the learning effect was present in two-thirds of the listeners, for nonsense speech with noise, it was present in half of them, and for meaningful speech without noise, it was present in one-third, despite initial training. It was not found for meaningful speech with noise. The learning effect for meaningful speech without intervening noise was always very small; for nonsense speech, it could cause the score to rise from $40 \%$ up to as much as $80 \%$. However, if a learning effect were correlated with the inclination of the regression line, we would expect to find the individual regression lines (improvement of intelligibility by noise vs. intelligibility without noise) to be steeper for lists that were presented later; we did not find this to be the case, and therefore reject the learning effect as an explanation.

\section{Effect of Noise on Intelligibility}

Our results clearly show that intervening noise improves the intelligibility of interrupted speech both for meaningful and nonsense speech, although the scores and the distribution of scores differ for the two kinds of speech. The disagreement in the literature on the point of improved intelligibility with intervening noise cannot be attributed to differences in speech material (redundancy level). The explanation for the conflicting results has to be found in some other factor. A probable one is the experience of the observers in psychoacoustic testing. First, we have to understand that Miller and Licklider trained their listeners. They report that training resulted in an improvement of speech intelligibility of interrupted speech without intervening noise. We did not train our listeners extensively, and a close look at our results shows that, for some listeners, performance with noise was quite similar to that without noise; these listeners were experienced in psychophysical testing. The less experienced listeners show marked differences in results, with intervening noise always yielding better intelligibility. This suggests that a difference in training or motivation may be responsible for the difference in behavior.

In this regard, it is worthwhile to note that the variance in the scores is smaller when intervening noise is added than when no noise is added. It seems that observers performing well without intervening noise make maximum use of the speech information and thus since no additional speech information is available can perform no better with intervening noise. Those performing poorly without intervening noise do not make maximum use of the available speech information in this condition, but do so if the noise is switched on. It seems as if the noise makes them switch over to another way of performing. Such performance is better established for meaningful speech than for the unfamiliar nonsense speech.

We chose a $-15-\mathrm{dB}$ signal-to-noise ratio in the experiments, because a very good continuity effect could be achieved at this ratio. Pilot studies had shown that full continuity perception is found for ratios below $-10 \mathrm{~dB}$, while intelligibility deteriorates below $-20 \mathrm{~dB}$, due to masking phenomena (Dirks \& Bower, 1970). It seems unlikely to be coincidental that Powers and Wilcox (1977) reported improved intelligibility with intervening noise in the range from -6 to $-24 \mathrm{~dB}-$ very close to the above-mentioned limits determined by us. This supports the idea that improved intelligibility is found only under conditions in which continuity is obtained.

\section{Mechanism Underlying Improvement of Speech Intelligibility with Noise}

An explanation for the better intelligibility of interrupted or alternated speech with intervening noise has been suggested by Huggins (1964). He noted that the waveform of a speech signal is distorted at moments of switching. This distortion may result in false articulation cues. Huggins suggested that noise could mask the distortions, eliminating false articulation cues and thus resulting in better intelligibility.

According to this view, the improvement in intelligibility is entirely due to differences in the peripheral processing. In our opinion, however, this explanation is not tenable in relation to our experimental results. The interrupted speech presented to our listeners was taped (gaps and all), so every listener received exactly the same information. Nevertheless, some listeners seemed to ignore all false cues, while others fell into every pitfall presented by the interruptions of the speech. Other arguments against a peripheral explanation can be found in the reduction of variability in intelligibility when intervening noise is added, in the differences in learning effects, and in the differences between nonsense and meaningful speech. All these facts point towards the involvement of more centrally located processes.

Listeners' reports of large perceptual differences between the conditions with and without intervening noise point in the same direction. Speech with intervening noise is perceived as being continuous, seems to be easier to follow, and is more intelligible than 
speech without noise. Most listeners seem to be tense while listening to interrupted speech without noise and to relax when noise is introduced.

These facts would seem to suggest that we process interrupted speech signals in different ways, depending on whether or not the gaps in the speech are filled with noise. We may hypothesize that, with intervening noise, some speech recognition process necessarily fills in the gaps in the signal, the redundancy level determining the possibilities offered to the auditory system in this filling-in process. Without the noise, listeners are aware of the gaps in information and seem to be forced to guess consciously what could have been said in them. The listener's performance, reflecting his or her motivation, vocabulary, intelligence, and experience, is of major importance then.

This process of unconsciously filling in missing speech information is something we experience often in daily life. Under normal conversational conditions, speech information is regularly masked by competing sounds such as coughs, banging doors, etc. We are conditioned to grasp the message without being bothered by the competing sounds-indeed, often without noticing them at all-but only if not even the slightest hint of a real interruption is present. The differences between meaningful and nonsense speech can be seen as resulting from differences in familiarity with the speech.

In a paper on segmented speech, Huggins (1975b) introduced the idea of "gap bridging." He noted that the introduction of silent intervals shorter than $65 \mathrm{msec}$ between the speech segments did not reduce intelligibility, although longer intervals did. On the basis of these findings, Huggins suggested that the auditory system can link speech segments across short gaps. Our speech material contains silent intervals longer than $65 \mathrm{msec}$ and is not segmented: contrary to Huggins's segmented speech, parts of the information in our material are really missing. A bridging effect such as that suggested by Huggins cannot take place. Nevertheless, we do seem to observe some kind of gap bridging in our interrupted speech samples with intervening noise, complemented by an interpolation mechanism (i.e., actual generation by the auditory system of a probable continuation of the observed speech signal to fill the gap). While the stimuli involved are-different, the mechanisms proposed by Huggins and us do seem to have considerable points of resemblance. It would be nice, in this connection, to see whether it is possible to extend Huggins's bridging effect by introducing noise into the silent intervals between his speech segments.

The unconscious interpolation mechanism suggested here can also explain the results of pulsation threshold measurements on interrupted music and pure tones described by Verschuure (1978). In that study, it was found that even transients or modulations could be perceived within a cut-out interval as long as the noise had a higher spectral level at all frequencies and hints of the events were present in the parts of the signal that were not cut out. In all instances, the brain seems to construct the most likely percept to fill the gaps. If no noise was inserted in the gaps, this process did not take place. With our length of interval, the process occurs only if continuity is perceived, which occurs if the absence of the signal in the silent interval cannot be detected.

The findings described in this paper and by Powers and Wilcox have their consequences for the explanation of the continuity effect on which pulsation threshold measurement is based. Verschuure (1978, $1981 \mathrm{~b}$ ) formulated a working hypothesis for the analysis of pulsation patterns very similar to that presented by Houtgast (1974). Primarily designed to explain cochlear filtering, this hypothesis stated that continuity was perceived if no information indicating that a signal had been interrupted was available to any channel of the auditory system. The information in question was defined as a significant drop in the activity of the particular channel. Taking the findings described in the present paper into account, we can rephrase this hypothesis to include peripheral and central processes as follows:

When an acoustic signal is temporarily interrupted and another signal is presented during the (short) interruption period, we perceive the interruption only if the activity in some auditory channel drops by a significant amount during the interruption. If the interruption is not perceived, the brain interprets the activity as resulting not from an alternation of signals, but from a simultaneous presentation of a weaker continuous signal and an interrupted louder signal. The gap appearing in the signal perceived as continuous is filled with the most probable signal, generated within the auditory system itself.

The first part of this hypothesis now deals with cochlear analysis, the second with the mechanism of signal interpretation, and the third with the unconscious interpolation process. The hypothesis covers not only regular interruptions, but also the irregular short ones so common in daily life.

\section{Conclusions}

The insertion of noise in the silent intervals of interrupted speech leads to better intelligibility.

The intelligibility of interrupted speech with intervening noise depends on the redundancy level of the speech.

The improvement of intelligibility by intervening noise is primarily determined by the performance of the listener in the absence of noise. 
Differences in speech material are not the reason for the controversy in the literature as to whether or not added noise does lead to a real increase in intelligibility. A probable reason is the difference in experience the listeners have had in psychoacoustic testing.

There is a relation between improved intelligibility with noise and the continuity effect.

Intervening noise seems to allow the auditory system to perceive interrupted signals as if they were continuous, automatically filling the gaps with the most probable signal. This occurs if no cue for the absence of the signal is perceived.

\section{REFERENCES}

Bocca, E., \& Calearo, C. Central hearing processes. In J. Jerger (Ed.), Modern developments in audiology. New York: Academic Press, 1963.

Cherry, E. C. Some experiments on the recognition of speech, with one and with two ears. Journal of the Acoustical Society of America, 1953, 25, 975-979.

Cherry, E. C., \& Taylor, W. K. Some further experiments upon the recognition of speech, with one and with two ears. Journal of the Acoustical Society of America, 1954, 26, 554-559.

Cherky, E. C., \& Wiley, R. Speech communication in very noisy environments. Nature, 1967, 214, 1164.

Dirks, D. D., \& Bower, D. Effect of forward and backward masking on speech intelligibility. Journal of the Acoustical Society of America, 1970, 47, 1003-1007.

Hopxinson, N. T. Combined effects of interruption and interaural alternation on speech intelligibility. Language and Speech, $1967,10,234-243$.

Houtoast, T. Psychophysical evidence for lateral inhibition in hearing. Journal of the Acoustical Society of America, 1972, 51, 1885-1894.

Houtgast, T. Lateral suppression in hearing (Doctoral dissertation, Free University Amsterdam). Amsterdam: Academische pers, 1974.

Hugains, A. W. F. Distortion of the temporal pattern of speech: Interruption and alternation. Journal of the Acoustical Society of America, 1964, 36, 1055-1064.

Hugarss, A. W. F. Temporally segmented speech and "echoic" storage. In A. Cohen \& S. O. Nooteboom (Eds.), Structure and process in speech perception. New York: Springer, 1975. (a)
Hugains, A. W. F. Temporally segmented speech. Perception \& Psychophysics, 1975, 18, 149-157. (b)

Huares, G. W., Houge, A. S., Goldstein, D. P., \& Rupf, J. A. Is the intelligibility of alternated speech related to rate or place of alternation? Journal of the Acoustical Society of America, $1966,40,1283-7 \mathrm{H} 9$.

KREUL, E. J. Speech intelligibility for interaural alternated speech with and without intervening noise for words and nonsense. Language and Speech, 1971, 14, 99-107.

Marks, L. E., \& Miller, G. A. The role of semantic and syntactic constraints in the memorization of English sentences. Journal of Verbal Learning and Verbal Behavior, 1964, 3, 1-5.

MrLlen, G. A., \& Lickliden, J. C. R. The intelligibility of interrupted speech. Journal of the Acoustical Society of America, 1950, 22, 167-173.

Powers, G. L., \& Speaks, C. Intelligibility of temporally interrupted speech. Journal of the Acoustical Society of America, 1973, 54, 661-667.

Powers, G. L., \& Wilcox, J. C. Intelligibility of temporally interrupted speech with and without intervening noise. Journal of the Acoustical Society of America, 1977, 61, 195-199.

Schubert, E. D., \& Parker, C. D. Addition to Cherry's finding on switching speech between the two ears. Journal of the Acoustical Society of America, 1955, 27, 792-794.

Sigael, S. Nonparametric statistics for the behavioral sciences. Tokyo: McOraw-Hill Kogakusha, 1956.

Spenks, C., \& Trooren, T. T. Interaural alternation and speech intelligibility. Journal of the Acoustical Society of America, $1974,56,640-644$.

VerschuUre, J. Pulsation threshold patterns and neurophysiological tuning. In E. F. Evans \& J. P. Wilson (Eds.), Psychophysics and physiology of hearing. London: Academic Press, 1977.

VerschuUre, J. Auditory excitation patterns (Doctoral dissertation, Erasmus University Rotterdam). Delft: W. D. Meinema, 1978.

VerschuURe, J. Pulsation patterns and nonlinearity of auditory tuning. I. Psychophysical results. Acustica, 1981, 49, 288-295. (a)

VerschuUre, J. Pulsation patterns and nonlinearity of auditory tuning. II. Analysis of psychophysical results. Acustica, 1981, 49, 296-306. (b)

Verschuure, J., Rodenbura, M., \& Maas, A. J. J. Presentation conditions of the pulsation threshold method. Acustica, $1976,35,47-54$.

WingField, A., \& Wheale, J. L. Interaural alternation, information load, and speech intelligibility. Journal of the Acoustical Society of America, 1975, 57, 1219-1220.

(Manuscript received June 1, 1982; revision accepted for publication December 3, 1982.) 